\title{
Sistema remoto para la medición y visualización del consumo de agua
}

https://doi.org/10.53358/ideas.v3i1.554

Sebastián Bahamonde ${ }^{1}$, Ana Zambrano ${ }^{1}$, Ricardo Rosero ${ }^{2}$

${ }^{1}$ Ingeniería Eléctrica y Electrónica, Escuela Politécnica Nacional; Quito, Ecuador

${ }^{2}$ Instituto Superior Tecnológico Sucre, Carrera de Electricidad, Avenida 10 de agosto N26-27 y Luis Mosquera Narváez-Ecuador 170109

Quito, Ecuador

${ }^{1}\left\{j o r g e . b a h a m o n d e\right.$, ana.zambrano\}@epn.edu.ec, ${ }^{2}$ rrosero@tecnologicosucre.edu.ec

\section{RESUMEN}

El presente trabajo propone un sistema de distribución para leer y visualizar el consumo de agua de manera remota, por medio de una página web y un aplicativo móvil. Para la implementación del sistema se lo ha dividido en tres etapas que son: módulo electrónico, módulo web y módulo móvil, y, para su desarrollo se ha establecido una arquitectura de tres niveles: presentación, aplicación y datos. Cada uno de estos niveles se encuentran alojados en el Cloud Computing de Microsoft Azure. El módulo electrónico se encarga de medir y registrar el consumo de agua y los datos obtenidos se guardan en una base de datos, el módulo web se encarga de gestionar los usuarios y administradores, permite las consultas sobre el consumo de agua de los diferentes controladores y en el módulo móvil se encuentra un APK que puede ser instalado en un dispositivo móvil para consultar el consumo de agua de los diferentes controladores.

Palabras Claves: Medidor Remoto, APK, Consumo de Agua, Microsoft Azure

Abstract. This document proposes a distribution system to be able to read and view water consumption remotely, through a web page and a mobile application. For the implementation of the system, it has been divided into three stages which are: electronic module, web module and mobile module and for its development a three-level architecture (presentation, application and data) has been established. Each of these levels are hosted in Microsoft Azure Cloud Computing. The electronic module is in charge of measuring and recording water consumption and the data obtained is stored in a database, the web module is in charge of managing users and administrators, it allows queries about the water consumption of the different controllers and in the mobile module there is an APK that can be installed on a mobile device to be able to consult the water consumption of the different controllers.

Keywords: Remote Meter, APK, Water Consumption, Microsoft Azure 


\section{Introducción}

El uso de servicios por medio de las redes de comunicación, en los últimos años ha aumentado considerablemente, debido a las ventajas de accesibilidad permanente y la reducción de tiempo, en el caso de la realización de un trámite o la consulta de un servicio, sin la necesidad de que el usuario tenga que desplazarse hacia la institución para poder realizar algún servicio. Actualmente en el Ecuador, el servicio de agua potable no se ha visto beneficiado del uso de nuevas tecnologías y sistemas de comunicación; la toma del valor del consumo de agua potable se lo realiza manualmente en cada una de las viviendas.

El uso de sistemas distribuidos para la lectura del consumo de agua se lo está empleando en Chile, donde ya se han hecho pruebas de funcionamiento de este tipo de sistemas con NB-loT [1] y medidores Kamstrup [2]. Es por eso que, en el país es necesario modernizar estos servicios y establecer un sistema que permita la recolección de datos del consumo de agua potable con las tecnologías e infraestructura que se tiene actualmente, por lo que, se va a desarrollar un sistema de distribución para la medición de consumo de agua y visualización de información.

El sistema se encuentra dividido en tres etapas. La primera etapa, está conformado por la parte sistema electrónico que realiza la medición del consumo de agua potable mediante un sensor de flujo que se encuentra conectado a una placa de Arduino WENOS D1, la que contiene una conexión Wifi para el enlace, con alguna red local para el envío de información hacia el internet, un lector de tarjeta Micro SD y un circuito de alimentación; la información del sensor de caudal es almacenada en una base de tatos. La segunda etapa está conformada por el aplicativo web para la administración del sistema, donde se podrá crear, registrar, actualizar y eliminar a los clientes y medidores; además, de poder observar en tiempo real el consumo de agua de los clientes. La tercera etapa se compone del diseño e implementación del aplicativo móvil, en la misma que, el cliente pueda realizar consultas sobre el consumo del o los medidores que se encuentren vinculados. El valor de consumo se almacenará una vez al día en un horario específico, y si no existe una conexión hacia internet en el horario establecido para el almacenamiento de éste, se almacenará en 122 una memoria externa con la fecha de cuando se realizó la medición. Este valor o valores 
serán enviados y almacenados en la base de datos cuando exista conexión a internet en el horario específico.

\section{Fundamento Teórico}

\section{Estado del arte de sistemas de medición de agua potable}

El desarrollo de las comunicaciones electrónicas ha permitido realizar el envío de datos entre sistemas, haciendo uso de diferentes tipos de medios; estos datos pueden ser: voltajes, velocidades, flujos, presiones, temperaturas entre otros.

En la actualidad existen sistemas de medición remotas que hacen uso de radio frecuencias, telefonía celular e internet. Con los sistemas de medición remota se llega a tener información de diferentes equipos en tiempo real como: perfiles de consumo, fugas, daños en instrumentos de medición, entre otros.

Además, si estos sistemas son aplicados a tareas como la medición del consumo de agua potable, el usuario se asegura de conocer lo que realmente ha consumido [3].

Entre las soluciones actuales para la medición del consumo de agua remotamente, existen soluciones que hacen uso de nuevas tecnologías de comunicación como es el caso de BMETERS [4], la cual implementa la tecnología de comunicación LoRaWan. El funcionamiento de esta solución se basa en un módulo de radio, agregado a un medidor de consumo de agua que posee una salida de pulsos eléctricos; el módulo de radio es el encargado de realizar la conexión a la red y de enviar el valor de consumo del usuario para ser almacenado [4].

Otra solución a la medición remota del consumo de agua potable, la entrega Gignac Energie [5], que consta de un sensor iPERL sensor de medición con tecnología magnética, que es un sistema de comunicación por radio llamado SensusRF, el cual opera en una banda sin licencia y un portal web para tener acceso a los datos llamado H2Olmes. El funcionamiento de esta solución se basa en el sistema de comunicación SensusRF, que consta de dos partes: la una se encuentra en el medidor de agua y la otra, montado sobre un vehículo móvil. La parte del sistema de comunicación que se encuentra en el medidor de agua es la encargada de escuchar peticiones y enviar el valor de consumo de agua; por otro lado, la parte del sistema de comunicación que se encuentra en el vehículo móvil, es el encargado de solicitar y de receptar la información de los medidores. Además, posee una antena de telefonía móvil, encargada de realizar la conexión hacia internet para enviar la información hacia el servidor. Por la manera del funcionamiento del sistema SensusRF, el vehículo deberá desplazarse cerca de los medidores de agua para realizar la medición, puesto que, el alcance de la comunicación es máximo de un kilómetro [5].

El desarrollo de diferentes sistemas para realizar la medición remota de los medidores de agua dependerá de las tecnologías de comunicación usadas, así como también, de las necesidades y/o prestaciones que se tengan para implementarlas. En la actualidad, existen tecnologías de comunicación para realizar este tipo de mediciones como: Wireless M-Bus2, Sigfox3, LoRaWan, NB-loT4. Cada una de estas tecnologías tienen diferentes propósitos, como también, diferentes características, así: el alcance de la comunicación, la capacidad de transmitir datos con bajo consumo de energía, inmunidad a las interferencias con otros dispositivos, y otras.

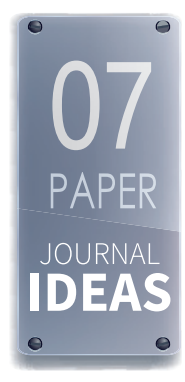




\section{Servicios web, aplicaciones web y móvil}

Son aplicaciones que se pueden escribir, publicar, localizar y buscar a través de una red y por su flexibilidad permiten integrar aplicaciones para la generación de redes de comunicaciones. Los servicios web permiten la interoperabilidad; lo que significa que, los servicios se pueden utilizar desde cualquier tipo de aplicación web o móvil, independientemente del lenguaje de programación en el que se haya desarrollado [6].

La comunicación que ofrece los servicios web entre aplicaciones se basa generalmente en dos tipos de arquitecturas: la arquitectura de Protocolo Simple de Acceso a Objetos - SOAP (Simple Object Access Protocol) y la arquitectura de Transferencia de Estado Representacional o REST (Representational State Transfer). [7].

\section{Cloud Computing}

El Cloud Computing es una tecnología que permite brindar una infraestructura informática, servicios o aplicaciones a cualquier usuario a través de internet. La nube es un conjunto de recursos virtuales como velocidad de procesamiento o memoria de almacenamiento, los cuales, se administran mediante un software de gestión y automatización. Para hacer uso de la nube o Cloud Computing es necesario sistemas operativos, software de virtualización, software de gestión y automatización. Los sistemas operativos hacen uso de las redes e interfaces del usuario host, el software de virtualización extrae todos los recursos y los agrupa en la nube, el software de automatización asigna los recursos y, por último, las herramientas de gestión proporcionan nuevos entornos [8].

Una nube pública es un depósito de recursos virtuales que son implementados a partir de un hardware, su propiedad es de una empresa ajena a la compañía o al usuario. En este tipo de nube, varias empresas pueden hacer uso de estos recursos, pero, de una manera separada; $y$, el proveedor es el encargado de realizar el mantenimiento y de la seguridad. Existen varios proveedores de nubes públicas, de los cuales, los más conocidos son AWS (Amazon Web Services), GoogleCloud, AlibabaCloud y Microsoft Azure.

Una nube privada es un grupo de recursos software y hardware, que se ofrecen a través del internet y que son de uso exclusivo para una empresa u organización. Este tipo de nubes se puede encontrar en el centro de datos de una empresa o alojarlas en proveedores externos, puesto que, a este, la define los ajustes de privacidad y las responsabilidades de gestión [9].

\section{Infraestructura como Servicio (laaS)}

Un servicio laaS es aquel, donde un proveedor brinda una infraestructura informática, posee un hardware y software mínimos requeridos para implementar una nube: la red, el almacenamiento, seguridad mediante firewalls, los servidores y la virtualización. El proveedor será el encargado de realizar la administración y el mantenimiento a estos recursos y el usuario puede hacer uso de estos, según lo requiera. Existen varias formas de uso para este tipo de servicio, que pueden ser: de manera temporal, para experimentación, alojamiento de proyectos web grandes, para dar solución al almacenamiento y copia de seguridad, análisis de big data, entre otros.

Una de las características más importantes para el usuario que hace uso de este tipo de servicio es la de realizar el pago al proveedor solo por los recursos que él está usando [10]. 


\section{Metodología}

\section{Estado actual del sistema de medición del consumo de agua}

El funcionamiento del sistema actual de medición y toma del valor de consumo de agua potable de los usuarios se muestra en la Fig. 1. Este sistema comienza por el medidor de agua potable del usuario, el cual tiene un flujómetro del tipo turbina tangencial. Este tipo de flujómetro es del tipo de "chorro único", lo que quiere decir que el caudal de entrada del flujómetro es desviado para incidir de forma tangencial sobre una turbina o hélice. Esta hélice se encuentra en sentido perpendicular al flujo del agua girando a una velocidad directamente proporcional al caudal volumétrico del líquido. El giro de la turbina o hélice hace que el contador mecánico se mueva y muestre la cantidad de consumo.

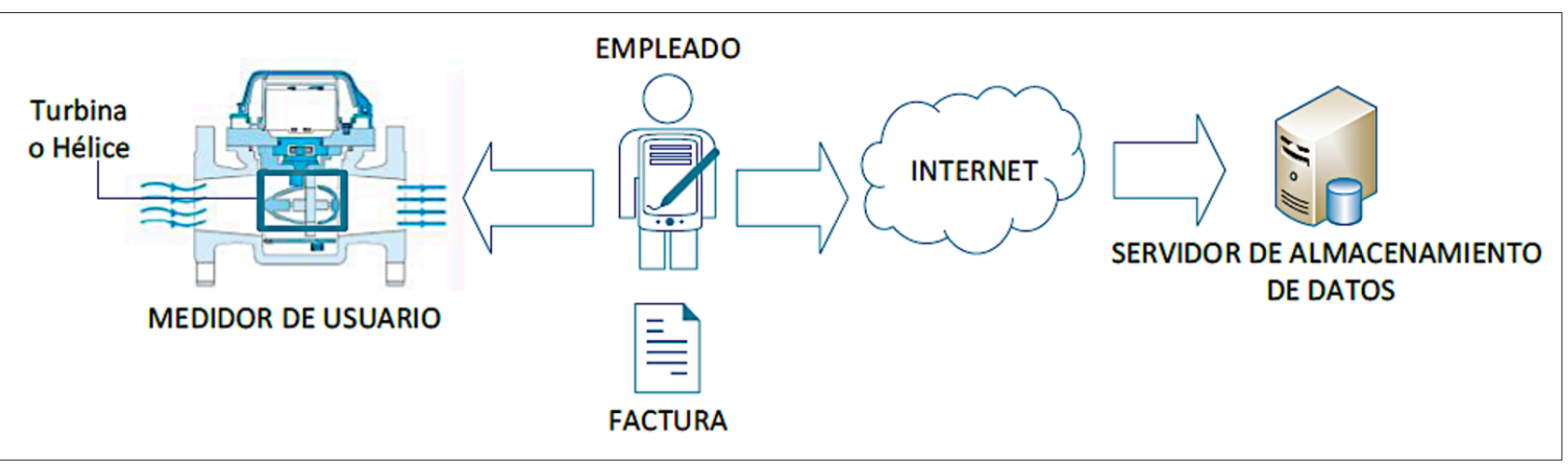

Fig. 1. Sistema actual de medición de agua potable.

Una vez que se tiene la forma de medir la cantidad de consumo de agua, por medio del personal contratado por la empresa se realiza la toma de los datos de manera manual en cada una de las casas por medio de la lectura de los medidores y registrando los datos mediante un dispositivo móvil diseñado para este propósito. Este proceso de toma del valor de consumo del agua potable se lo realiza una vez al mes por lo cual puede presentar diferentes problemas como error en la toma del valor de consumo por falla humana, fallo en la comunicación para el registro del valor de consumo y que éste no pueda ser ingresado.

\section{Tablero Kanban}

KanbanTool [11] es una herramienta en línea que sirve para realizar la gestión de tareas, pudiendo ser configuradas y modificadas con el fin de formar tableros Kanban. A cada tarea se le puede agregar la información necesaria para que el personal encargado pueda desarrollarla. En la Fig. 2 se muestra el Tablero Kanban correspondiente al diseño. 


\section{Lista Tareas}
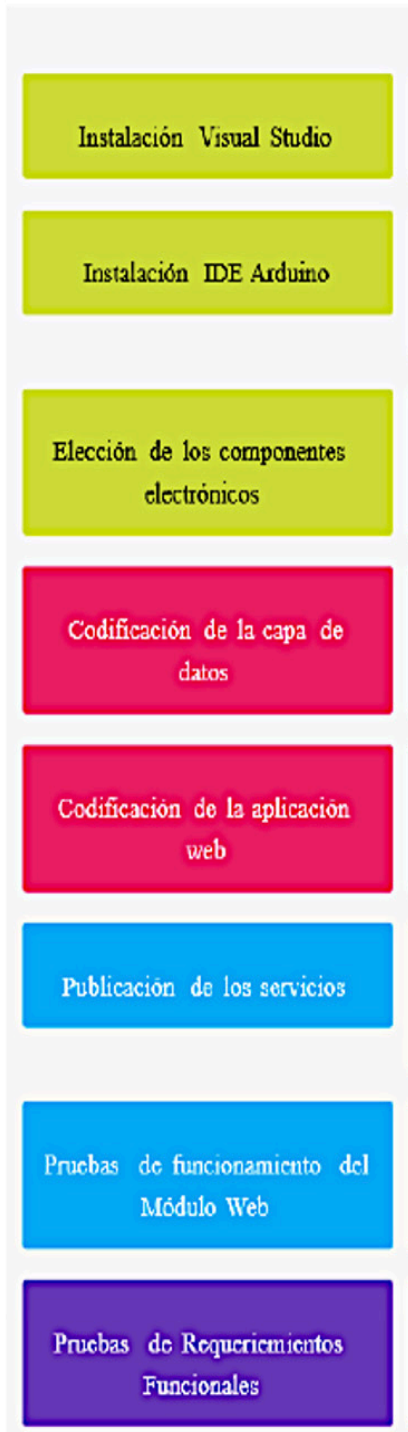

En Proceso

+ añadir tarca

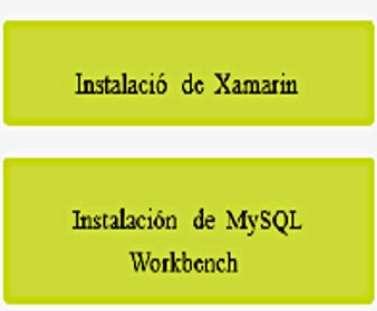

Codificación del Módulo Electrónico
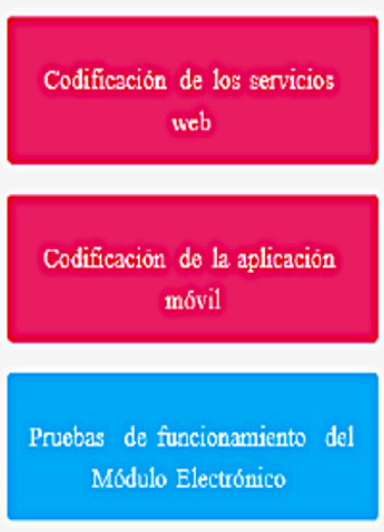

Prucbas de funcionamiento de la Módulo Móvil
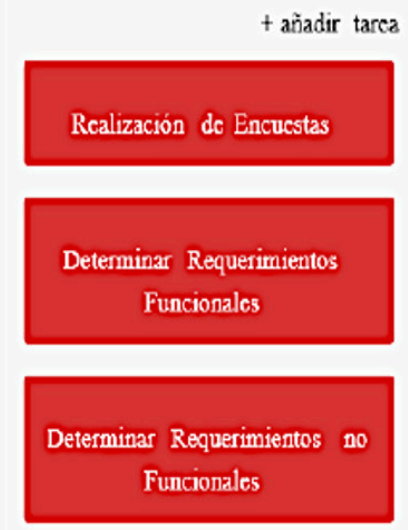

Diseño del diagrama de flujo para Módulo Electrónico

Diseño de la capa de datos

Disefio de la capa de negocio

Diseño de la capa de presentación
Completadas

+ añadir tarca

El tablero de la Fig. 2 consta de tres columnas las cuales son: "Lista Tareas", "En Proceso" y "Completadas"; cada una de estas columnas representa el estado posible en el que se puede encontrar una tarea. En la columna "Lista Tareas" se encontrarán todas las tareas pendientes; en la columna "En Proceso" se encontrarán las tareas que se encuentren realizándose y por último en la columna "Completadas" se encontrarán las tareas que se encuentran terminadas.

El tablero Kanban de la Fig. 3 muestra las tareas para la fase de implementación que se deben realizar para el cumplimiento de los requerimientos funcionales de los tres módulos. 
Lista Tareas

+ añadir tarea
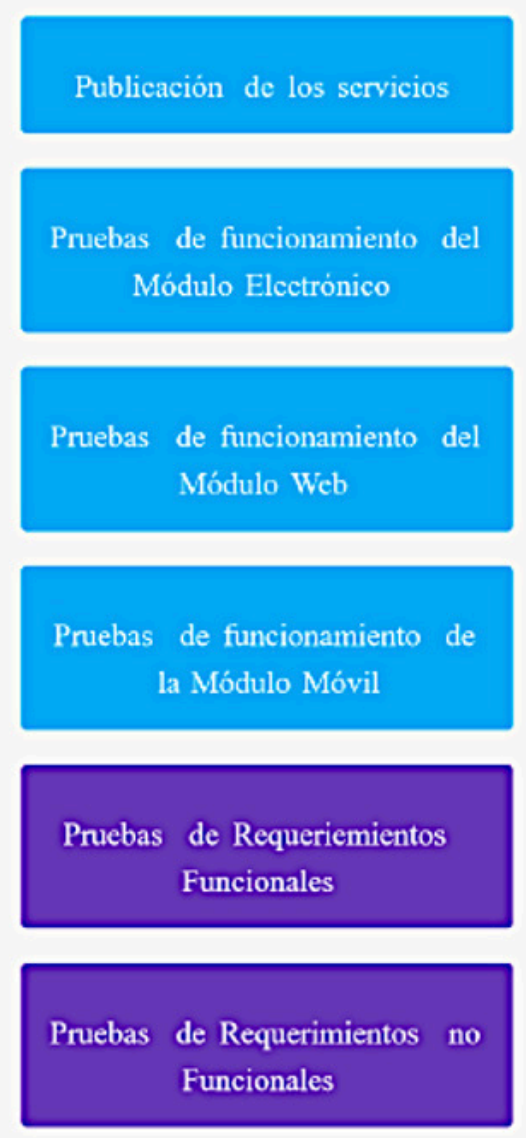
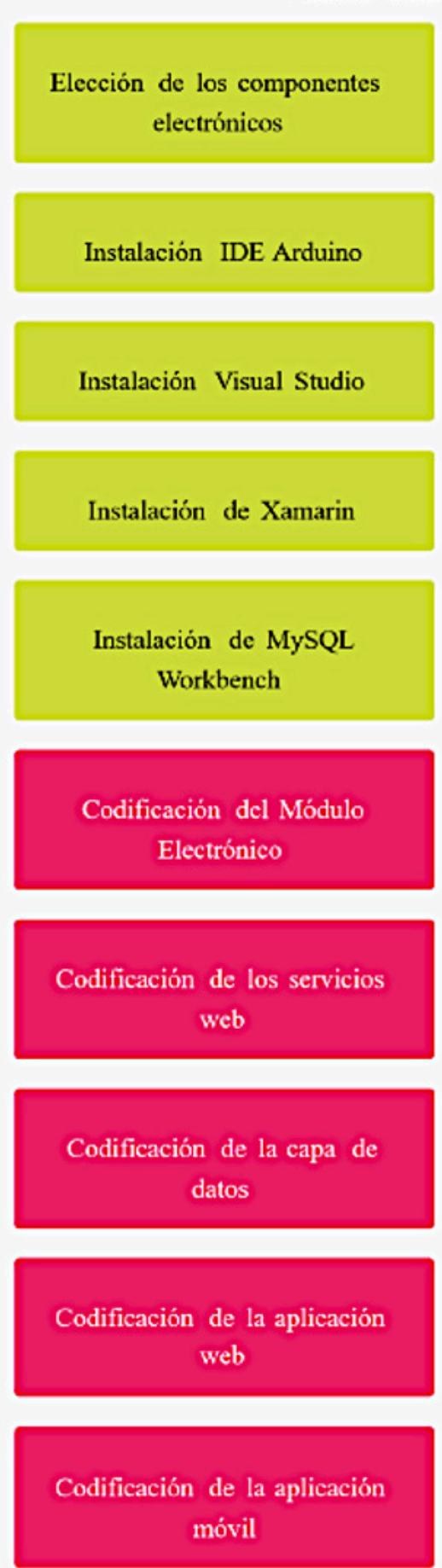

\section{Completadas}

+ añadir tarea
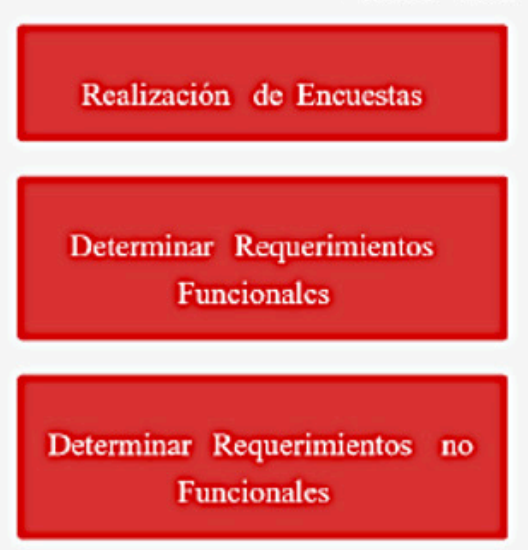

Diseño del diagrama de flujo para Módulo Electrónico

Diseño de la capa de datos

Diseño de la capa de negocio

Diseño de la capa de presentación

Fig. 3. Tablero Kanban implementación.

\section{Funcionamiento global del prototipo}

Se representa el funcionamiento del prototipo para el sistema de medición del consumo de agua de manera remota. El prototipo consta de tres módulos: Módulo Electrónico, Módulo Web y Módulo Móvil. 


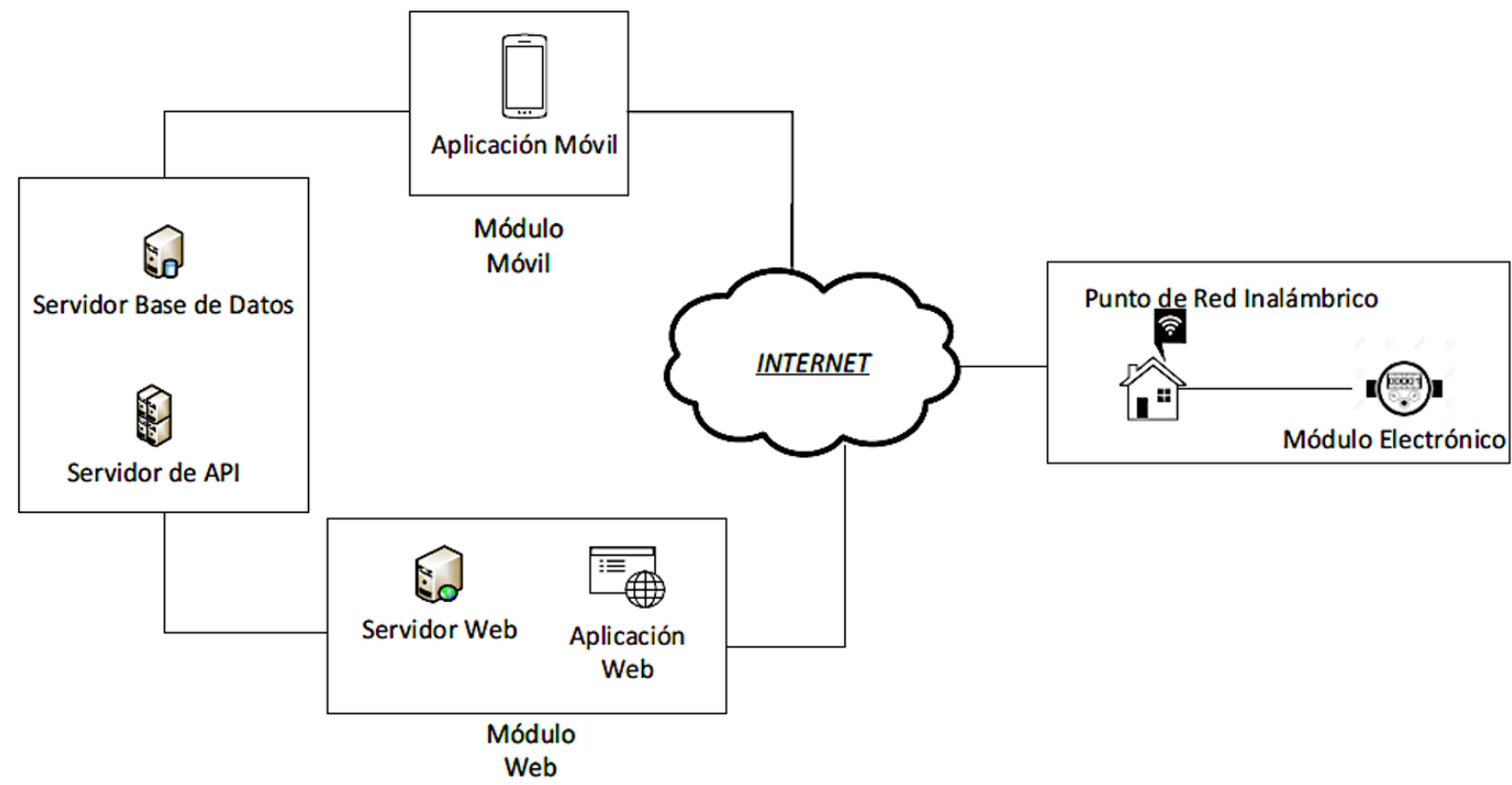

Fig. 4. Diagrama de funcionamiento global del prototipo.

Para la generación del sistema distribuido, el prototipo consta de tres tipos de servidores: Servidor Web, Servidor API y un Servidor de Bases de Datos; estos servidores forman parte de cada uno de los módulos.

El Módulo Electrónico, dentro del sistema será el encargado de realizar la medición del consumo de agua potable del usuario. Este módulo hará uso de los Servidores de API y Base de Datos para el almacenamiento de los valores de consumo.

Por otro lado, el Módulo Web dentro del sistema tiene el papel de gestionar a los usuarios, medidores y administradores; por lo cual este módulo tiene una aplicación web, la cual será alojada en un servidor web para que sea accesible desde cualquier lugar. Además, este módulo hará el uso de los Servidores API y Base de Datos para obtener, almacenar, eliminar o editar información.

Por último, se tiene el Módulo Móvil, el cual consta de una aplicación móvil que se deberá instalar en un Smartphone con el sistema operativo Android. Esta aplicación hará el uso de los Servidores de API y Base de Datos para realizar consultas de consumo de agua potable de un medidor en específico.

\section{Diseño de los módulos del prototipo}

\section{Módulo electrónico}

Para el módulo electrónico se determinarán los componentes necesarios para el funcionamiento de la parte de hardware y el diseño del software. El hardware de este módulo se encuentra compuesto de varios componentes los cuales se muestran en la Fig. 5. 


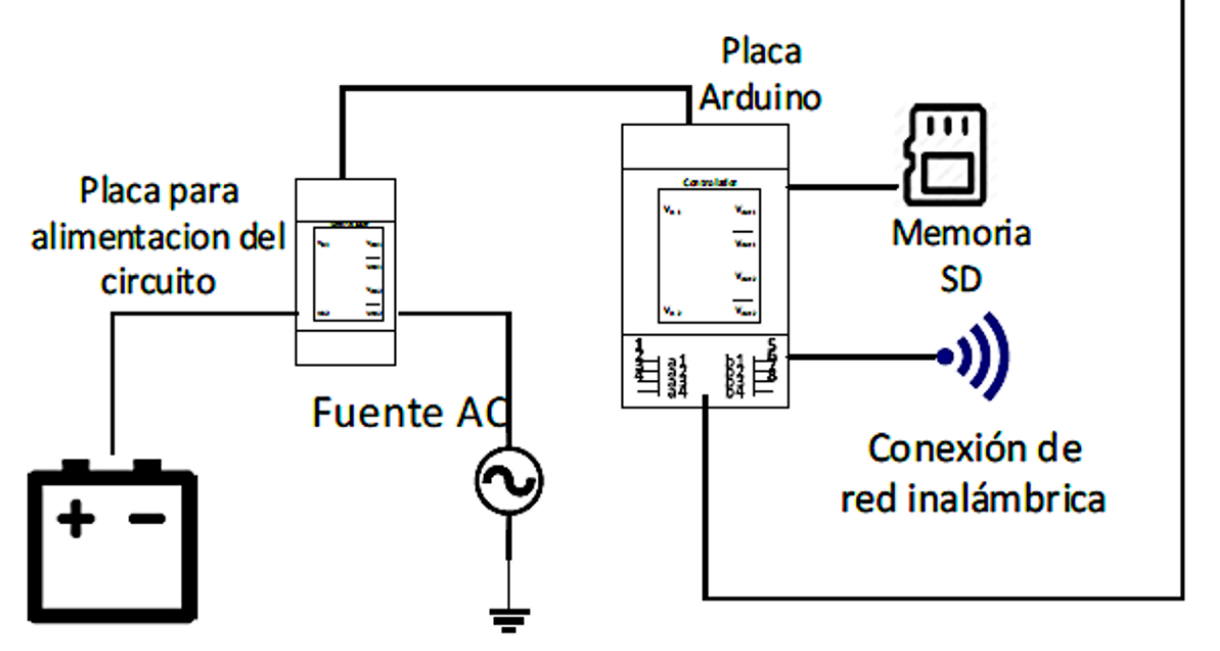

Caudalímetro o Flujómetro

Batería

Fig. 5. Módulo Electrónico.

Cada uno de estos elementos cumplen un propósito específico dentro del módulo electrónico.

Tabla 1. Elementos del módulo electrónico.

\begin{tabular}{|l|l|}
\hline \multicolumn{1}{|c|}{ Elemento } & \multicolumn{1}{c|}{ Función } \\
\hline $\begin{array}{l}\text { Placa de } \\
\text { Alimentación del } \\
\text { Circuito }\end{array}$ & $\begin{array}{l}\text { Encargada de realizar la alimentación al circuito y } \\
\text { de realizar el intercambio entre la energía normal y } \\
\text { la energía de la batería; además recarga la batería } \\
\text { cuando exista la presencia de la energía normal. }\end{array}$ \\
\hline Placa Arduino & $\begin{array}{l}\text { Esta placa junto con el chip ESP8266, será la } \\
\text { encargada de tener la lógica de funcionamiento de } \\
\text { todo el medidor electrónico. }\end{array}$ \\
\hline Flujómetro & $\begin{array}{l}\text { Este elemento será el encargado de producir pulsos } \\
\text { eléctricos para que sean leídos y procesados por la } \\
\text { placa Arduino con el fin de obtener el consumo de } \\
\text { agua utilizado. }\end{array}$ \\
\hline Memoria SD & $\begin{array}{l}\text { Encargado de almacenar el valor de consumo } \\
\text { cuando no exista conexión hacia internet. }\end{array}$ \\
\hline Batería & Encargada de dar respaldo de energía al medidor. \\
\hline
\end{tabular}

El sensor de flujo o caudalímetro YF-S201 tiene una válvula plástica, un rotor y un sensor de efecto hall; y es usado para líquidos de baja viscosidad como: bebidas gasificadas, bebidas alcohólicas, combustible, etc. El caudalímetro es compatible con la mayoría de los sistemas digitales como Arduino, Circuito Integrado Programable o PIC, Raspberry Pi, Controlador Lógico Programable, etc. [12].

El funcionamiento del caudalímetro empieza con el flujo del agua a través del rotor haciéndolo girar a diferentes velocidades, el rotor se encuentra unido a un imán el cual activa al sensor de efecto hall, generando un pulso eléctrico el cual puede ser leído por una entrada digital. 
Para calcular el volumen del líquido que pasa a través del flujómetro se debe conocer el factor de conversión $\mathrm{K}$ de frecuencia a caudal el cual será diferente dependiendo del diámetro de la tubería. El fabricante especifica tres ecuaciones para el cálculo del volumen del líquido que pasa por el flujómetro. Estas ecuaciones se las detallan a continuación [40].

La Ecuación uno, es usada para calcular el caudal a partir de la frecuencia en un flujómetro YF-S201

$$
f(H z)=K * Q \frac{L}{\min }
$$

Una vez calculado el caudal que pasa a través del flujómetro se podrá calcular el volumen con la ecuación dos:

$$
V=V o+Q \Delta t
$$

El valor de K dará la exactitud en la medida del flujómetro, por lo cual si se requiere una mayor exactitud en la medición se deberá realizar el cálculo del factor de conversión con la Ecuación 3.

$$
K=\frac{N^{\circ} \text { Pulsos }}{\text { Volumen } * 60}
$$

Para la programación de los módulos se presentan los siguientes diagramas de flujo, en los que se representa de manera gráfica el algoritmo del proceso de la programación estructurada, en la Fig. 6 se representa el proceso principal del funcionamiento del sistema, en el cual existen las etiquetas $[A, B, C]$ las cuales representan a funciones cuyo algoritmo de funcionamiento se lo detallará en distintos diagramas de flujo.

La etiqueta " $A$ " dentro de la Fig. 6 representa al algoritmo para el ingreso del valor de consumo de agua potable en la base de datos; este algoritmo se encuentra detallado en la Fig. 7. La etiqueta " $B$ " dentro de la Fig. 6 representa el algoritmo para almacenar en la memoria SD el valor de consumo que no se haya podido ingresar en la base de datos debido a que no existe conexión a internet, no se ha conectado a una red inalámbrica o si el servicio web para el almacenamiento de valor de consumo no se encuentra disponible durante lapso de una hora (que se encontrará configurado en el módulo electrónico para que realice el ingreso del valor de consumo). Este proceso se lo muestra en la Fig. 8. La etiqueta " $\mathrm{C}$ " dentro de la Fig. 6 representa el algoritmo para el ingreso de valores de consumo que hayan sido almacenados en la memoria SD del medidor debido a la falta de conexión hacia internet; este proceso se lo muestra en la Fig. 9. 


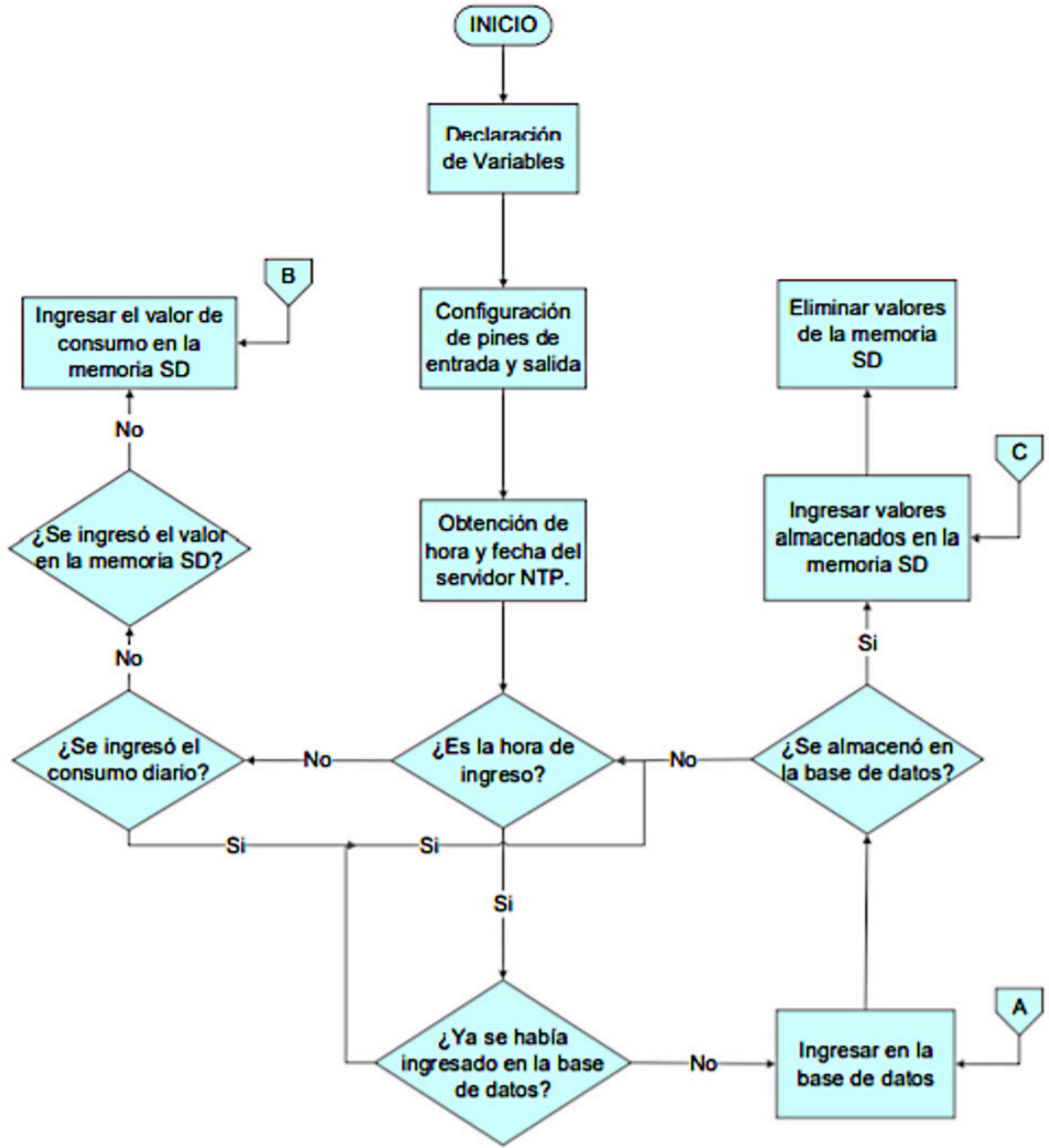

Fig. 6. Algoritmo principal del medidor electrónico. 


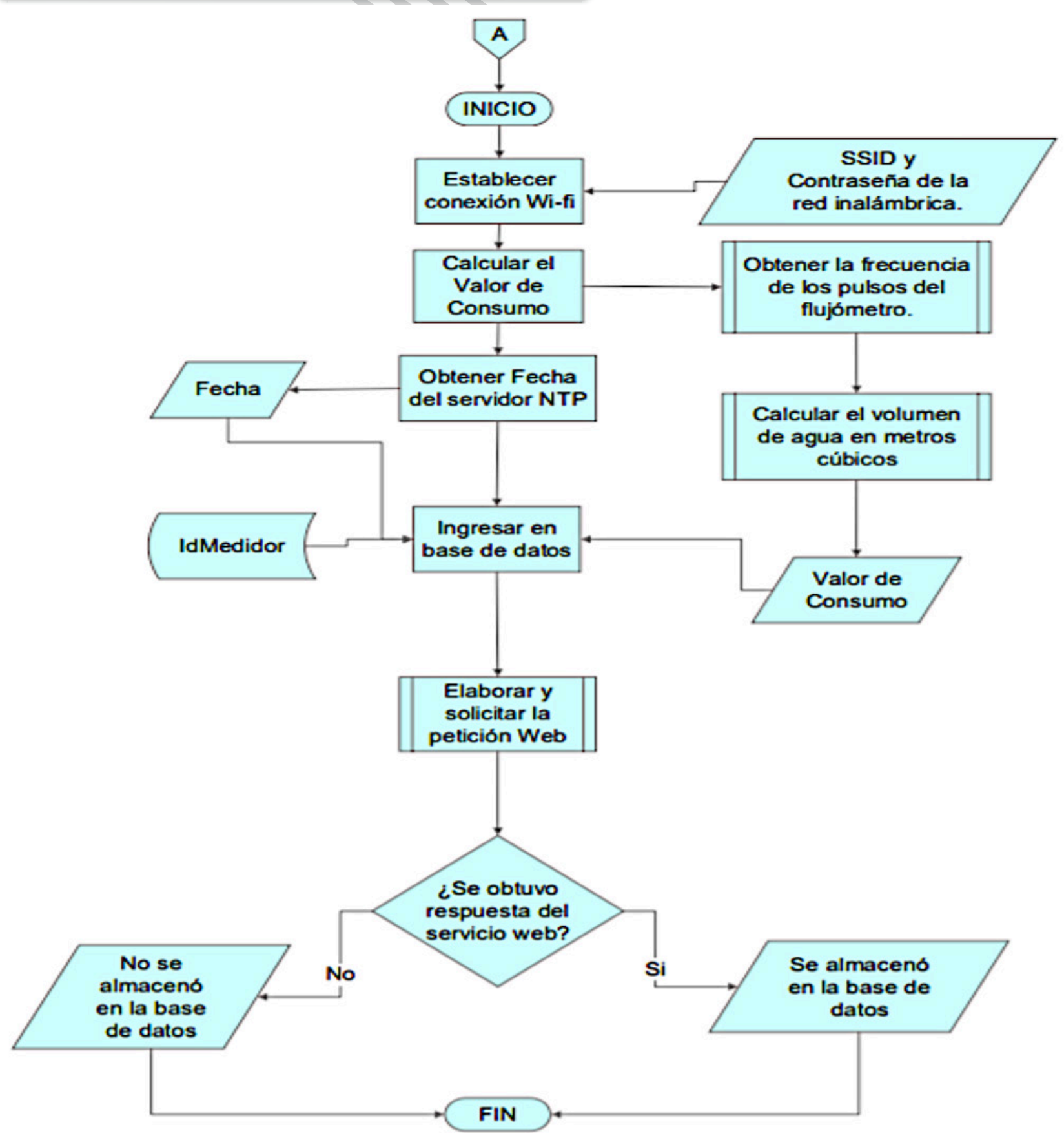

Fig. 7. Algoritmo de ingreso a la base de datos del valor de consumo. 


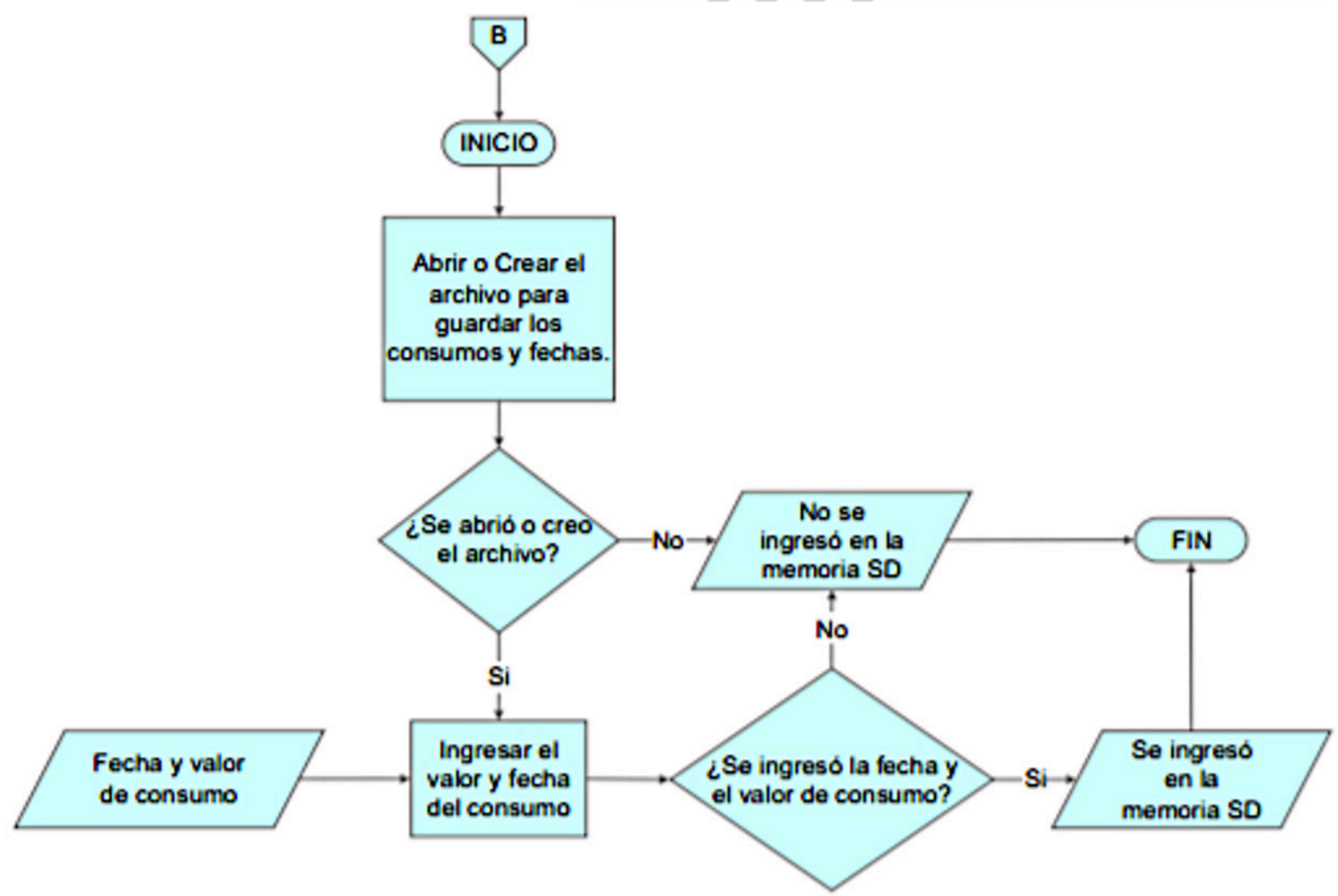

Fig. 8. Algoritmo de ingreso de valores a la memoria SD.

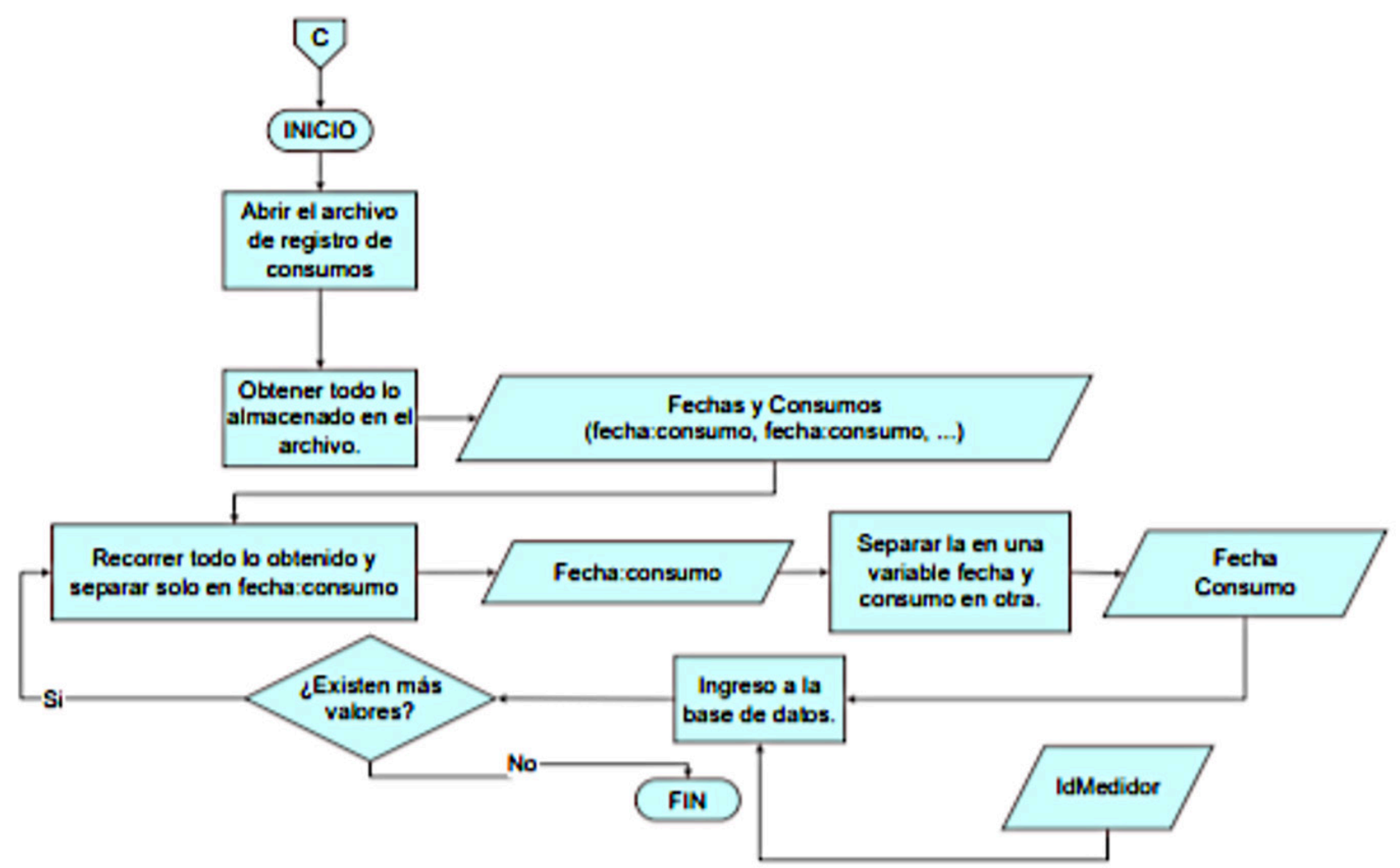

Fig. 9. Algoritmo de ingreso a la base de datos de valores de consumo atrasados. 


\section{Diseño de la capa de datos}

La capa de datos es representada a través del diseño de un diagrama relacional entre entidades. El diseño de la base de datos MySOL se muestra en la Fig. 10. Se tiene cuatro entidades que intervendrán en el almacenamiento de datos. Cada una de estas entidades poseen campos, entre los cuales están las claves primarias y claves foráneas; las claves primarias son atributos con valores únicos en cada entidad y las claves foráneas son usadas para la relación entre las entidades.

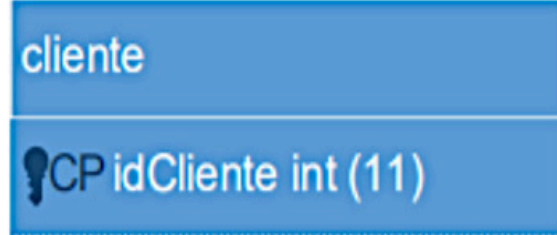

nombres varchar(45)

apellidos varchar(45)

mail varchar(45)

cédula varchar(45)

teléfono varchar(45)

\section{administrador \\ fCPidAdministradorint(11)}

Apellidos varchar(45)

Nombres varchar(45)

UserName

$\operatorname{varchar(45)}$

Password varchar(45)

\section{medidor}

fCP idMedidor in(11)

dirección varchar(45)

sector varchar(45)

serieMedidor

$\operatorname{varchar}(45)$

fFK idCliente int(11)

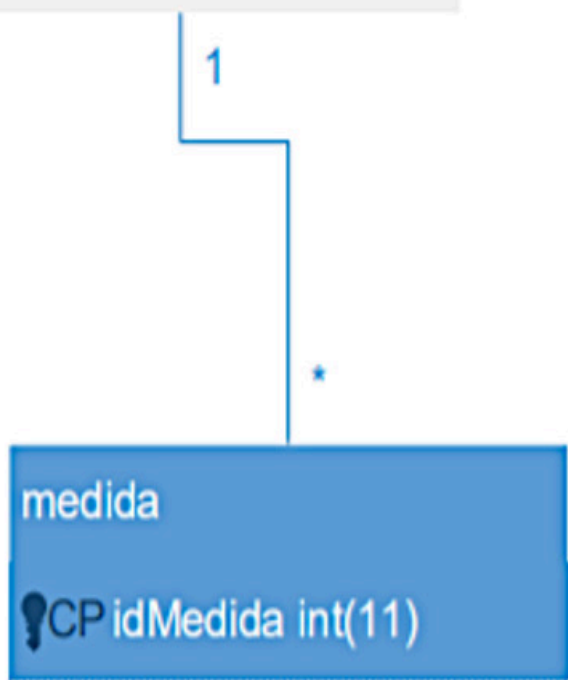

consumo varchar(45)

fechaMedicion

varchar(45)

fFK idMedidor int(11)

Fig. 10. Diagrama relacional entre entidades. 
Por ejemplo, la entidad de medidor posee campos: ubicación (dirección, sector), identificación (idMedidor, serieMedidor) y la clave foránea (idCliente). Esta clave foránea se la tiene debido a que un cliente puede tener varios medidores, por lo cual, existe una cardinalidad de "uno a muchos" entre la entidad Cliente y Medidor.

Tabla 2. Descripción de la entidad medidor.

\begin{tabular}{|l|l|l|}
\hline \multicolumn{1}{|c|}{ CAMPO } & \multicolumn{1}{|c|}{ TIPO } & \multicolumn{1}{c|}{ DESCRIPCIÓN } \\
\hline idMedidor & Número Entero & $\begin{array}{l}\text { Campo usado como identificador único } \\
\text { dentro del sistema para cada medidor. }\end{array}$ \\
\hline Dirección & $\begin{array}{l}\text { Cadena de } \\
\text { caracteres }\end{array}$ & $\begin{array}{l}\text { Campo usado para determinar la dirección } \\
\text { donde se encuentra ubicado el medidor. }\end{array}$ \\
\hline Sector & $\begin{array}{l}\text { Cadena de } \\
\text { caracteres }\end{array}$ & $\begin{array}{l}\text { Campo usado para determinar el sector } \\
\text { específico donde se encuentra el medidor, } \\
\text { este campo se requiere ya que la dirección } \\
\text { puede repetirse. }\end{array}$ \\
\hline serieMedidor & $\begin{array}{l}\text { Cadena de } \\
\text { caracteres }\end{array}$ & $\begin{array}{l}\text { Campo usado como identificador único del } \\
\text { medidor para el cliente. }\end{array}$ \\
\hline idCliente & Número Entero & $\begin{array}{l}\text { Campo usado para determinar el cliente } \\
\text { propietario del medidor. }\end{array}$ \\
\hline
\end{tabular}

\section{Diseño de la capa de negocio}

En la capa de negocio se estable los diferentes diagramas del proceso para la programación de los diferentes servidores del sistema remoto para la medición y visualización del consumo de agua. Para definir cada uno de los procesos se hará el uso de diagramas de casos de uso, diagramas de clases y diagrama del servicio web.

\subsubsection{Diagramas de casos de uso}

En el diagrama de casos de uso, se establece la descripción de las acciones o funciones que provee el sistema desde el punto de vista del usuario. A través de este tipo de diagramas se detalla el papel que desempeñan los usuarios dentro del sistema remoto para la medición y visualización del consumo de agua.

- El sistema divide a los usuarios en cuatro tipos de actores los cuales son: Administrador, Cliente, Usuario Sistema y Usuario Medidor.

- El Administrador, es el encargado de realizar la gestión (creación, lectura, actualización o eliminación) de usuarios, medidores. También de poder realizar consultas de consumo de cada uno de los medidores del sistema. Este usuario hace uso del módulo web.

- El Cliente, es aquel que hace uso del módulo electrónico y del módulo móvil para medir o verificar el consumo de agua respectivamente.

- El Usuario Sistema, es aquel que se encargará de realizar consultas de consumo, calcular el consumo de los meses de cada medidor o medidores de cada uno de los usuarios para el envío de las notificaciones al cliente, a través de un correo electrónico el cual es enviado al final de cada mes. 
- El Usuario Medidor, es aquel que interviene en el sistema realizando la medición y el ingreso del consumo de agua en la base de datos o también lo almacena en una memoria SD si existen fallas en la conexión hacia internet, disponibilidad de los servicios web, etc.

Los Diagramas de Casos de Uso del sistema, involucran a todos los tipos de usuarios y todas las acciones que se pueden realizar dentro del sistema. A cada usuario se lo relaciona únicamente con las actividades que estos pueden realizar.

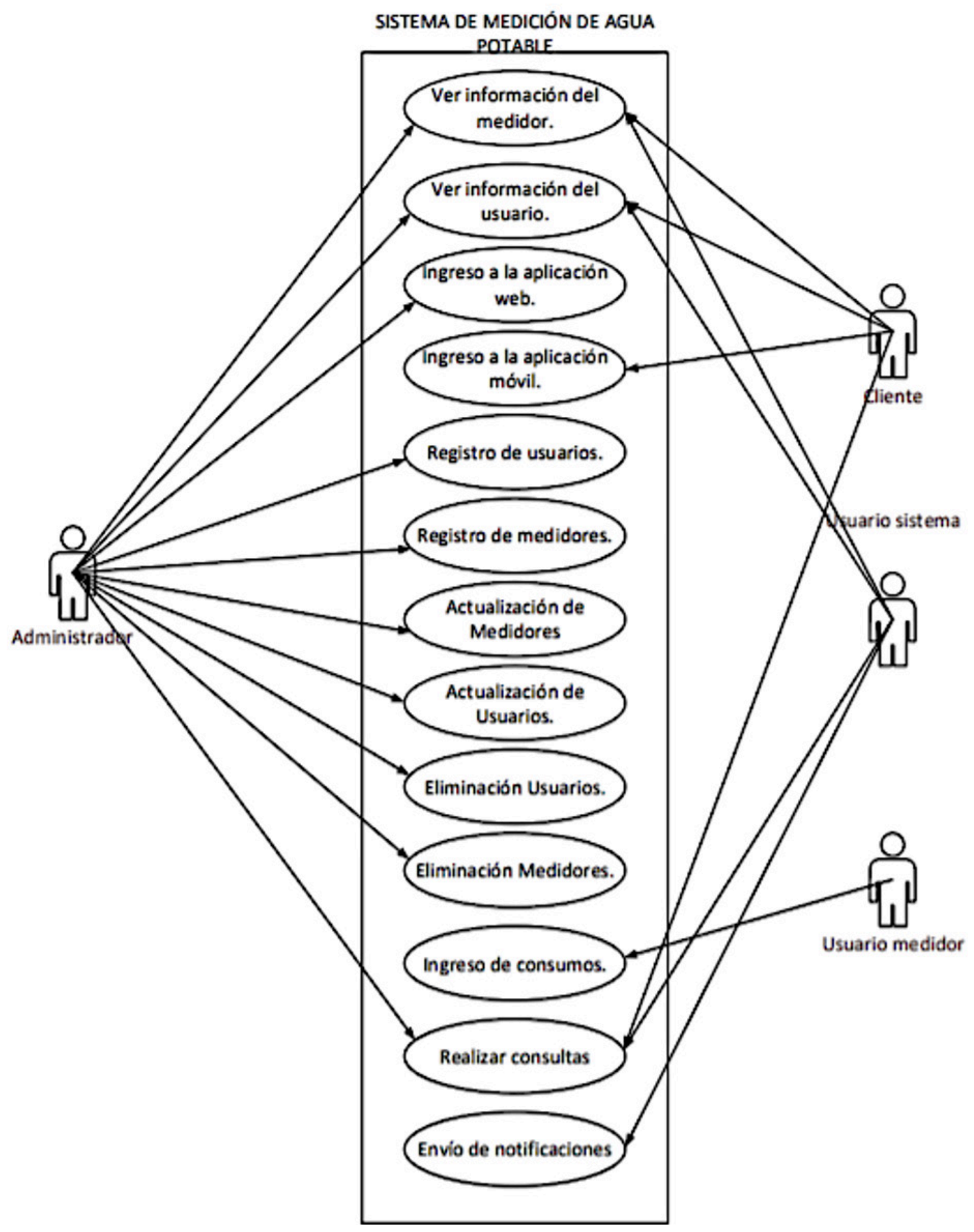

Fig. 11. Diagrama de casos de uso del sistema. 


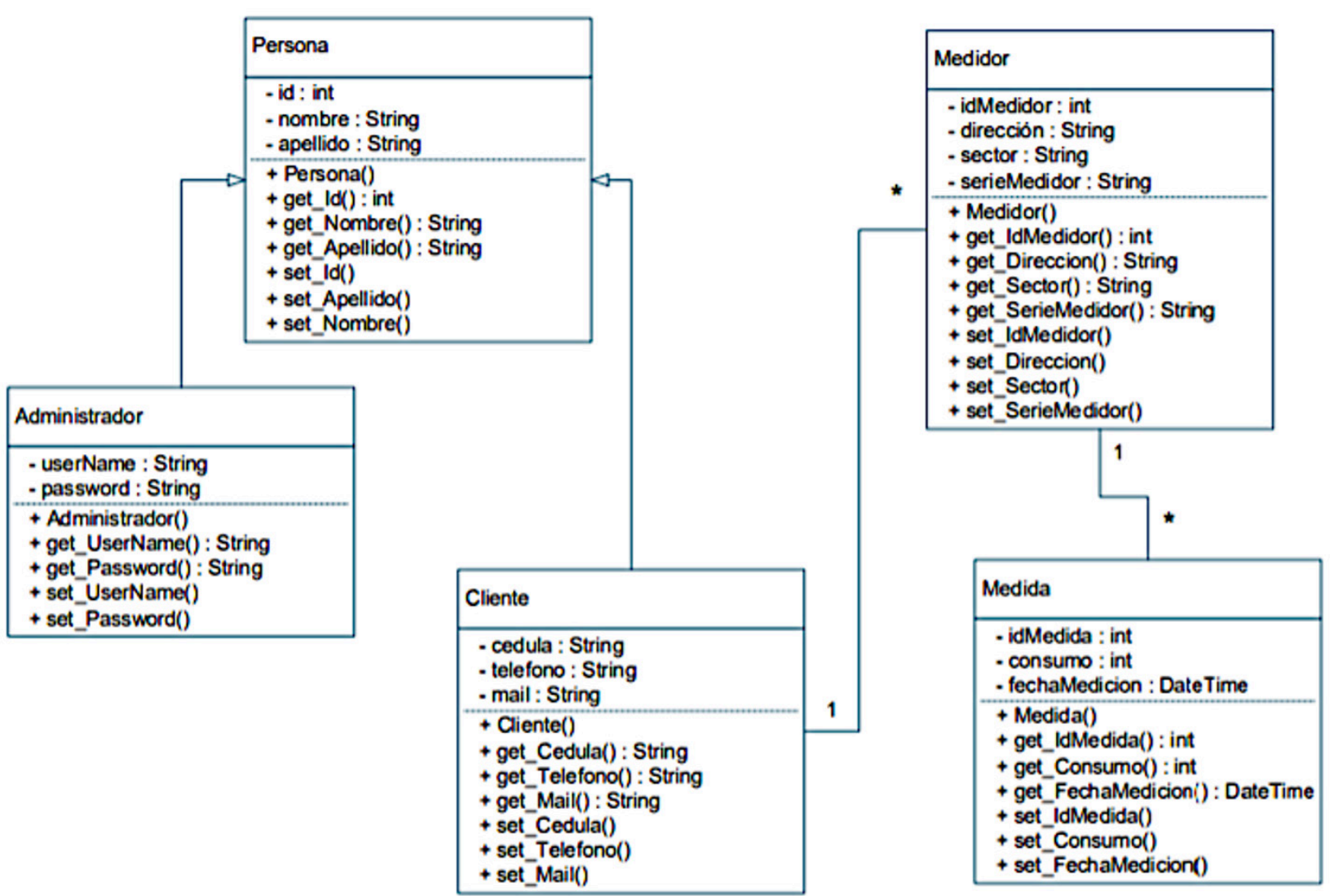

Fig. 12. Diagramas de clases del sistema.

Cada una de estas clases tienen sus propios atributos y métodos que son diferentes a las otras clases, por ejemplo, la clase Medidor posee atributos de diferentes tipos de datos y de acceso los cuales se detalla en la tabla 3. Además, se tiene los métodos "get" y "set" para cada uno de los atributos, los cuales servirán para conseguir y establecer su valor correspondiente.

Tabla 3. Descripción de la clase medidor.

\begin{tabular}{|l|l|l|l|}
\hline \multicolumn{1}{|c|}{ ATRIBUTO } & $\begin{array}{l}\text { TIPO DE } \\
\text { DATO }\end{array}$ & $\begin{array}{c}\text { TIPO DE } \\
\text { ACCESO }\end{array}$ & \multicolumn{1}{c|}{ DESCRIPCIÓN } \\
\hline idMedidor & $\begin{array}{l}\text { Numero } \\
\text { entero }\end{array}$ & Privado (-) & $\begin{array}{l}\text { Identificador único del medidor dentro del } \\
\text { sistema. }\end{array}$ \\
\hline Dirección & $\begin{array}{l}\text { Cadena de } \\
\text { caracteres }\end{array}$ & Privado (-) & $\begin{array}{l}\text { Atributo usado para identificar la dirección } \\
\text { de la ubicación del medidor. }\end{array}$ \\
\hline Sector & $\begin{array}{l}\text { Cadena de } \\
\text { caracteres }\end{array}$ & Privado (-) & $\begin{array}{l}\text { Atributo usado para identificar el sector de } \\
\text { la ubicación del medidor. }\end{array}$ \\
\hline serieMedidor & $\begin{array}{l}\text { Cadena de } \\
\text { caracteres }\end{array}$ & Privado (-) & $\begin{array}{l}\text { Identificador único del medidor para él } \\
\text { usuario. }\end{array}$ \\
\hline
\end{tabular}




\section{Diagrama del servicio web}

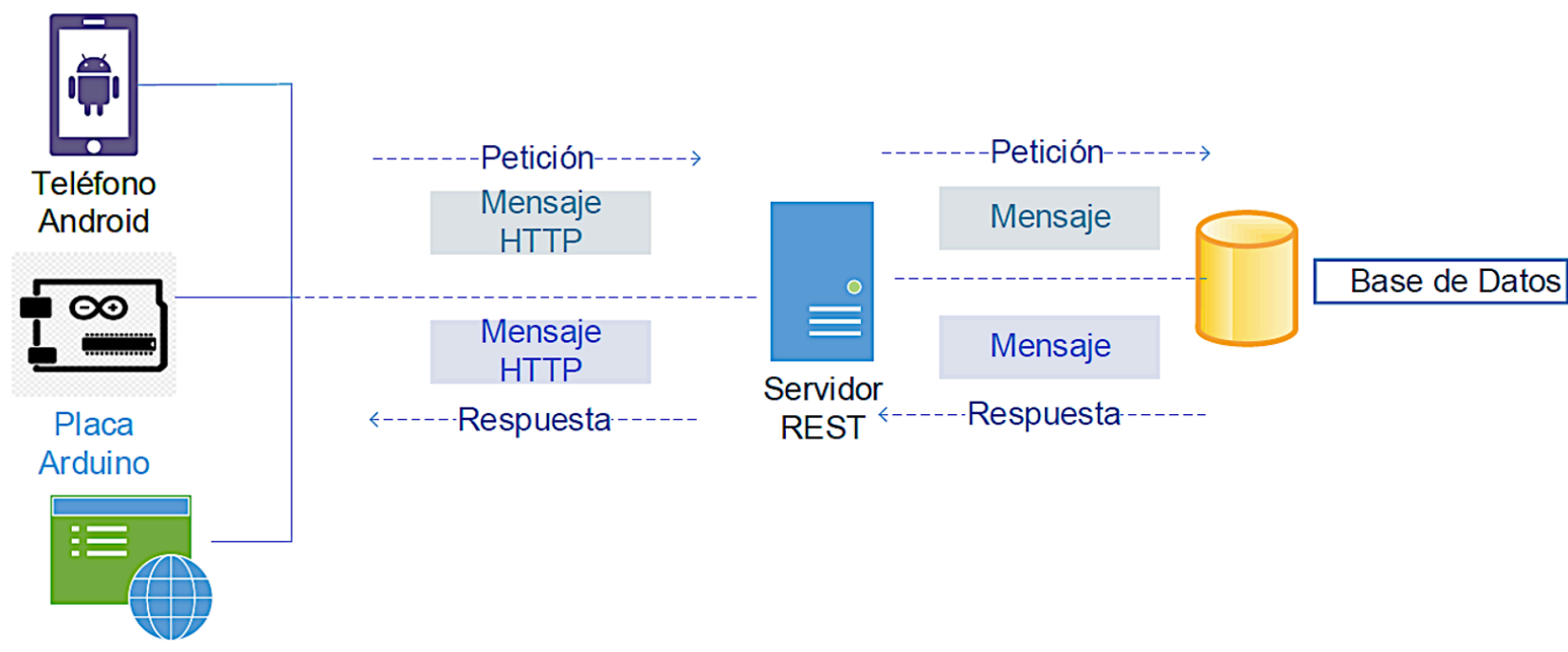

Aplicación Web

Los servicios web usados para el sistema de medición de consumo de agua son del tipo REST. Para el funcionamiento de este tipo de servidor el usuario deberá conocer la dirección IP del servidor o el nombre de dominio; además de conocer la estructura de las URIs para la solicitud de los diferentes servicios.

El funcionamiento del servicio tipo REST se presenta en la Fig.13.

Fig. 13. Funcionamiento de un servicio web.

Cada uno de los servicios web son diseñados siguiendo el funcionamiento de la Fig. 13. y estos contendrán las acciones necesarias para el cumplimiento de los requerimientos funcionales del sistema, por lo cual, cada servicio web realizará una acción (leer, escribir, editar o borrar) en la base de datos.

\section{Diseño de la capa de presentación}

En este apartado se detalla, el diseño de las interfaces de las aplicaciones web y móvil, por medio de las cuales el usuario podrá ingresar para saber el consumo del agua. Para lo cual se hará uso de la aplicación Balsamiq Wireframes, la cual es una herramienta que permite el diseño rápido de interfaces de usuario [13].

\section{Interfaz del módulo móvil}

El Módulo Móvil tiene cinco pantallas con el fin de cumplir las acciones establecidas para los requerimientos funcionales del sistema de medición del consumo de agua potable. Los bosquejos de la aplicación móvil se muestran en la Fig. 14.

138 Al inicio de la pantalla del aplicativo móvil muestra el ingreso del usuario y del número de 
serie del medidor el cual será único; con el objetivo de poder realizar la consulta de varios medidores de los cuales se tenga el número de serie.

La segunda pantalla, presenta la información básica del medidor y los últimos consumos que se han registrado en el sistema. Además, se podrá realizar consultas del consumo de

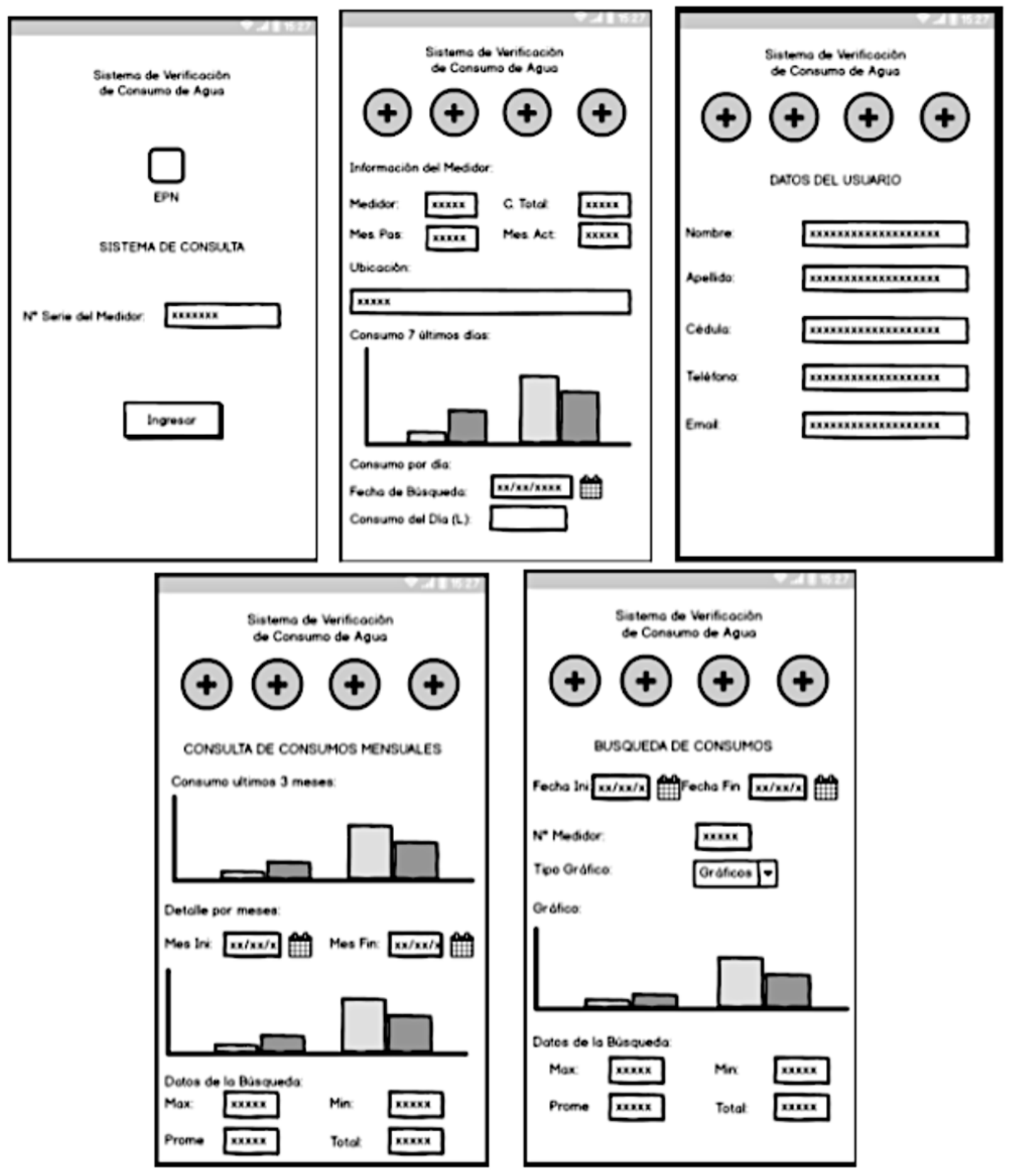

agua potable por día.

La tercera pantalla, presenta la información del cliente propietario del medidor.

En la cuarta pantalla, se puede realizar las consultas mensuales del consumo de agua del medidor y saber los valores de consumo máximo, mínimo, promedio y la suma total de los 139 
consumos realizados en los meses en los que se realice la consulta.

En la quinta pantalla, el usuario puede realizar las consultas del consumo de una fecha especifica y escoger el tipo de diagrama de presentación (Barchar, LineChart, PointChart, RadialGaugeChart, DonutChart, RadarChart) y además de observar valores de consumo máximo, mínimo, promedio y la suma total de los consumos de los días que se haya realizado la consulta.

Fig. 14. Interfaces módulo móvil.

\section{Interfaz del módulo web}

El módulo web no requiere de instalación, el usuario administrador puede hacer uso de esta aplicación a través de un navegador web, para lo cual el usuario debe conocer el nombre de dominio en donde se encuentre la aplicación. Una vez que el administrador ingrese el nombre del dominio o URL, ejemplo, "www.apliacionweb.com", se le presentará una página web en donde el administrador deberá realizar el ingreso de los parámetros requeridos para realizar la autenticación e ingreso al sistema. El bosquejo de la pantalla para realizar la autenticación del usuario se muestra en la Fig. 15.

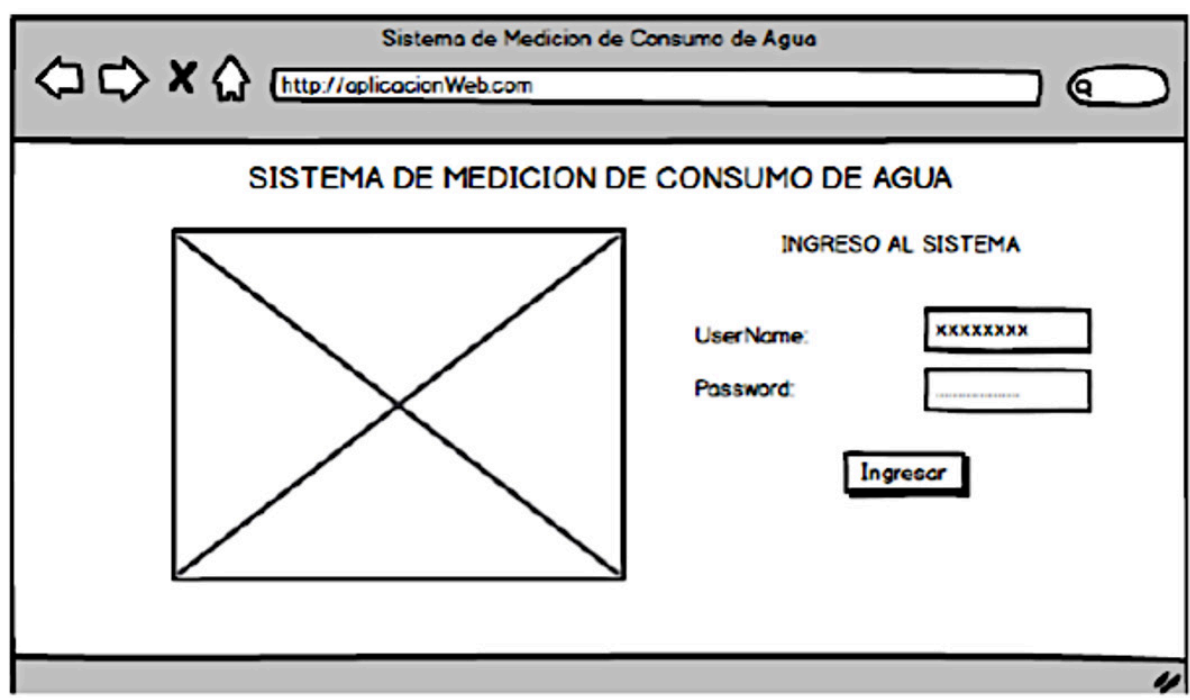

Fig. 15. Interfaz de ingreso a la aplicación web.

Desde esta interfaz el administrador podrá realizar la navegación a través del sistema mediante un panel situado en el lado izquierdo en donde se encuentra las diferentes opciones. Una de las opciones es "MEDIDORES" en donde se encuentra un menú de "Consultar" en la cual se podrá realizar la consulta del consumo de los distintos medidores registrados. Una vez se ingrese a esta opción se mostrará la interfaz de la Fig. 16. 
Lista Tareas
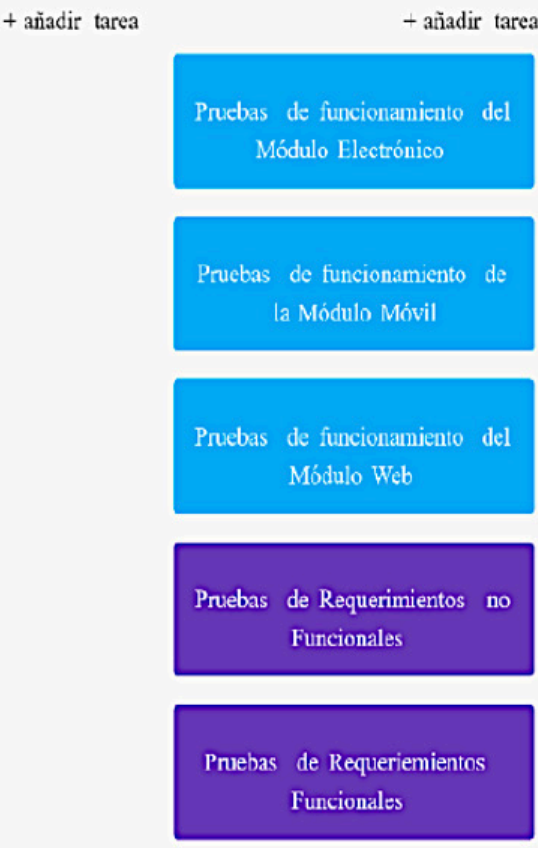

En Proceso

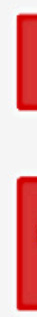

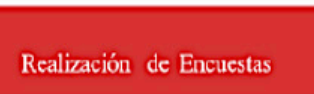

Realización de Encuestas

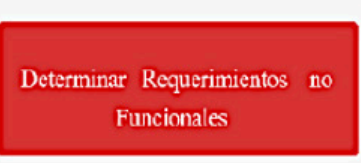

Diseño de la capa de datos
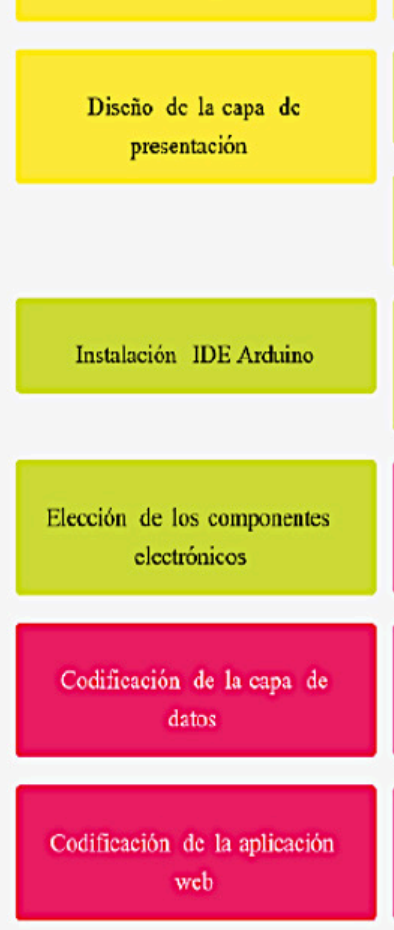

Publicación de los servicios
+ añadir tarea

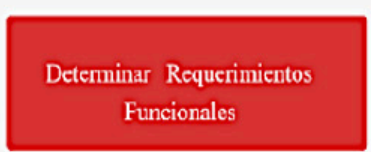

Diseño del diagrama de flujo para Módulo Flectrónico

Discrio de la capa de negocio

Instalación Visual Studio

Instalación de Xamarin

Instalación de MySQL Workbench

Codificación del Módulo Electrónico
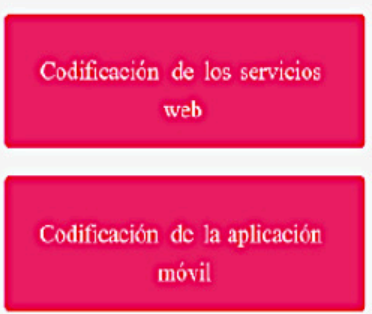


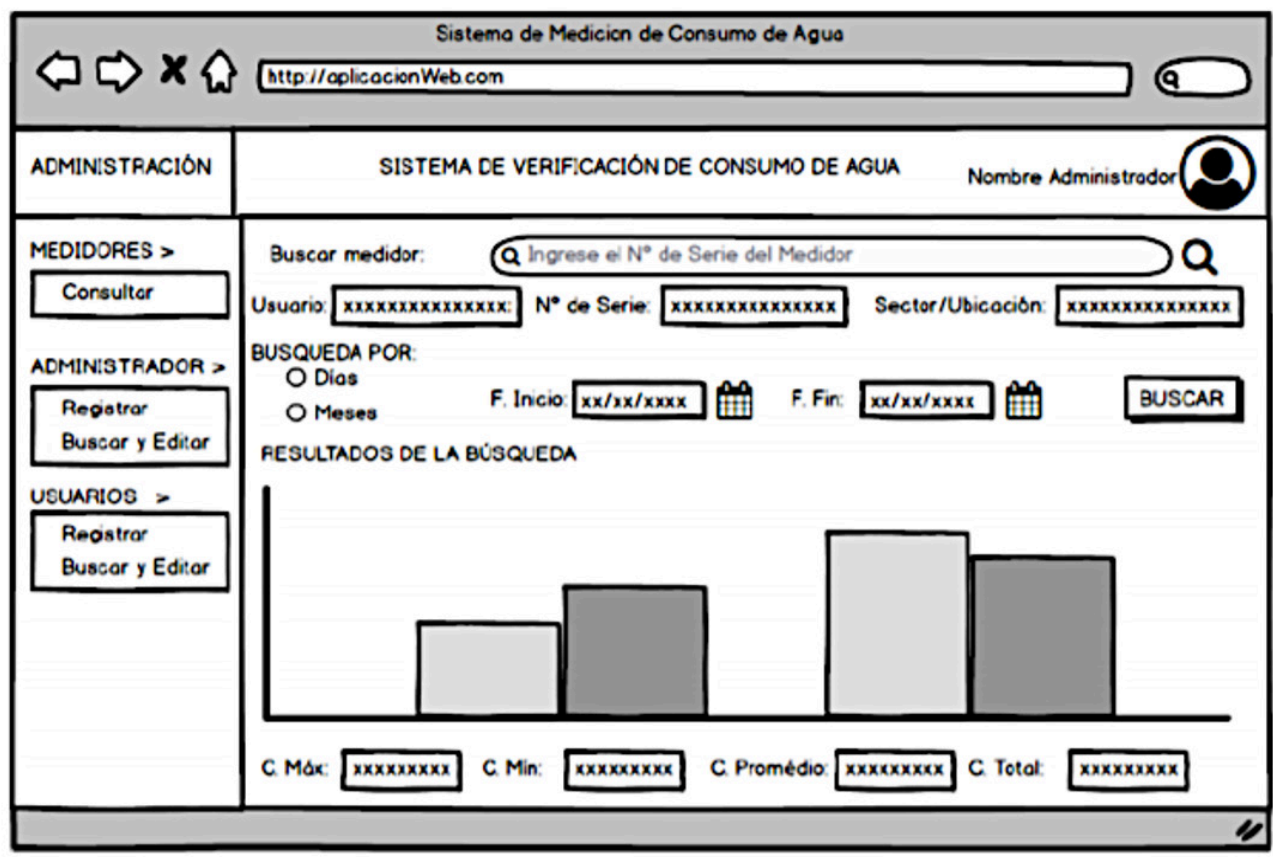

Fig. 16. Interfaz para realizar la consulta de consumos.

\section{Resultados y discusión}

Se realizaron diferentes pruebas a los distintos módulos que conforman el sistema, con el fin de determinar su correcto funcionamiento. Se llevó a cabo la actualización del tablero Kanban para la realización de las pruebas, como se muestra en la Fig. 17

Fig. 17. Tablero Kanban de pruebas.

Para la realización de las pruebas del módulo electrónico, se realizó la instalación del módulo en una casa y la verificación de su funcionamiento de cada una de las partes y sus requerimientos funcionales. En la Fig. 18 se muestra la instalación del módulo electrónico. 


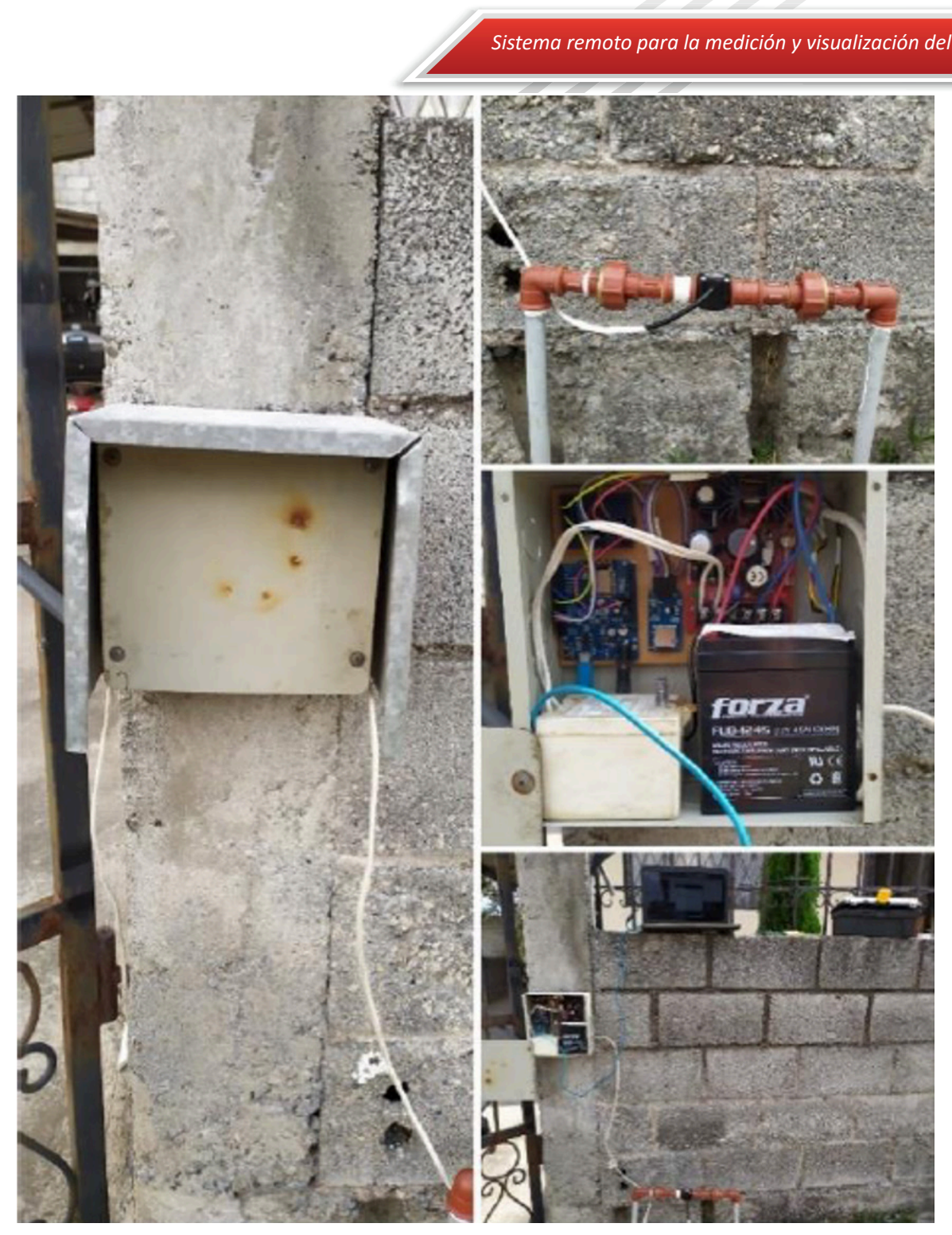

Fig. 18. Instalación del módulo electrónico

Los requerimientos funcionales del módulo electrónico se detallan en la tabla 4

Tabla 4. Requerimientos funcionales del módulo electrónico.

\begin{tabular}{|c|l|}
\hline $\begin{array}{c}\text { Requerimiento } \\
\text { funcional }\end{array}$ & \multicolumn{1}{c|}{ Descripción } \\
\hline RF-01 & $\begin{array}{l}\text { La placa Arduino debe ser capaz de conectarse } \\
\text { a la red inalámbrica del usuario. }\end{array}$ \\
\hline RF-02 & $\begin{array}{l}\text { Debe realizar el ingreso del valor de consumo a } \\
\text { la base de datos. }\end{array}$ \\
\hline RF-01 & $\begin{array}{l}\text { El valor de consumo debe ser almacenado en } \\
\text { una memoria SD si no existe conexión a internet } \\
\text { para el ingreso en la base de datos. }\end{array}$ \\
\hline RF-04 & $\begin{array}{l}\text { El medidor debe funcionar de manera } \\
\text { ininterrumpida. }\end{array}$ \\
\hline
\end{tabular}

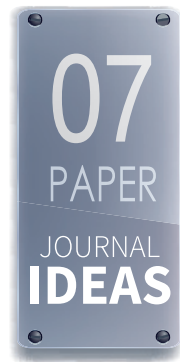


El módulo electrónico tiene un funcionamiento correcto al momento de almacenar en la memoria microSD o en la base de datos, sin embargo, existen diferencias entre los valores obtenidos por el prototipo y el medidor de agua actual. Esto puede ser debido a la apreciación que se tuvo al momento de observar el valor en el medidor de agua actual, pero concluidas las mediciones, el margen de error se acerca al 0,98\%, por lo cual, las mediciones entre el prototipo y el medidor actual son semejantes.

Para establecer el correcto funcionamiento del módulo web, se realizaron las pruebas necesarias para el cumplimiento de los requerimientos funcionales que se encuentran en la tabla 5.

Tabla 5. Requerimientos funcionales del módulo web.

\begin{tabular}{|c|l|}
\hline $\begin{array}{c}\text { Requerimiento } \\
\text { funcional }\end{array}$ & \multicolumn{1}{|c|}{ Descripción } \\
\hline RF-05 & $\begin{array}{l}\text { Debe realizar una autenticación mediante un } \\
\text { nombre de usuario y contraseña. }\end{array}$ \\
\hline RF-06 & Debe permitir la administración de los usuarios. \\
\hline RF-07 & $\begin{array}{l}\text { Debe permitir la administración de los } \\
\text { medidores. }\end{array}$ \\
\hline RF-08 & $\begin{array}{l}\text { Debe permitir realizar consultas de consumos } \\
\text { de cada medidor. }\end{array}$ \\
\hline RF-09 & $\begin{array}{l}\text { Debe permitir el envío de notificaciones } \\
\text { mediante correos electrónicos. }\end{array}$ \\
\hline
\end{tabular}

Para establecer el correcto funcionamiento del módulo móvil, se realizaron las pruebas necesarias para el cumplimiento de los requerimientos funcionales que se encuentran en la tabla 6.

Tabla 6. Requerimientos funcionales del módulo móvil.

\begin{tabular}{|c|l|}
\hline $\begin{array}{c}\text { Requerimiento } \\
\text { funcional }\end{array}$ & \multicolumn{1}{|c|}{ Descripción } \\
\hline RF-10 & $\begin{array}{l}\text { Debe mostrar la información del usuario del } \\
\text { medidor. }\end{array}$ \\
\hline RF-11 & $\begin{array}{l}\text { Se debe realizar el ingreso a la aplicación móvil } \\
\text { mediante el número de serie del medidor. }\end{array}$ \\
\hline RF-12 & $\begin{array}{l}\text { Se debe mostrar el consumo total y consumo } \\
\text { de meses anteriores. }\end{array}$ \\
\hline RF-13 & $\begin{array}{l}\text { Debe realizar consultas de los consumos por } \\
\text { día, días y meses. }\end{array}$ \\
\hline
\end{tabular}

Por otro lado, los Módulos Móvil y Web que se presentaron a los usuarios disponen de una interfaz amigable y de fácil manejo. 


\section{Conclusiones}

El desarrollo del prototipo de un sistema remoto para la medición y visualización del consumo de agua es una manera innovadora y actual para la lectura del valor de consumo de agua. El prototipo consta de un módulo electrónico, módulo web y módulo móvil, los cuales, permiten realizar la lectura remota, la medición del valor de consumo de agua, la gestión del sistema y la ejecución de consultas de los valores de consumo de agua a un cliente de una manera sencilla.

Las tecnologías, herramientas y lenguajes de programación que se utilizaron para el desarrollo del prototipo se las eligió en base a los conocimientos referentes al desarrollo de software como: Bases de Datos Relacionales, Programación Orientada a Objetos y Aplicaciones Distribuidas. Las herramientas seleccionadas fueron: C\#, C, SQL como lenguajes de programación y MySQL Workbench, Visual Studio, Arduino IDE, Microsoft Azure.

El uso de la metodología ágil Kanban para el desarrollo del prototipo fue de gran utilidad, debido a que se logró organizar el trabajo en distintas tareas y conocer el estado en el que se encontraban en el desarrollo del prototipo en cualquier momento.

El diseño de cada uno de los módulos se lo realizó en base a los requerimientos funcionales y no funcionales que fueron adquiridos mediante encuestas realizadas a usuarios del sistema de medición de agua potable. Con estos requerimientos se procedió a realizar diagramas de flujo, diagramas de clases, diagramas de casos de uso, un diagrama EntidadRelación para la base de datos, con el fin de satisfacer los requerimientos obtenidos de las encuestas.

La implementación del prototipo se lo realizó siguiendo el diseño y el desarrollo por capas de software (datos, lógica y presentación); el mismo que, permite el funcionamiento del prototipo y se divida en tres distintas partes independientes, además, que puedan existir distintos grupos de desarrolladores y que cada uno de estos equipos trabajen en una sola capa sin afectar a los otros.

Los resultados adquiridos en la fase de pruebas de los requerimientos funcionales y no funcionales del prototipo determinaron que, el prototipo presenta un correcto funcionamiento. El módulo electrónico presenta un error en la medida de consumo, esto se debería a que el medidor mecánico actual presenta agujas analógicas para revisar el consumo realizado y por tal motivo se pudo haber cometido un error de apreciación del valor medido. Además, se estableció que las aplicaciones que se desarrollaron son amigables para el usuario, puesto que, presentan formularios claros para el ingreso de información, así como también, gráficos que facilitan al usuario el entendimiento de los datos que se muestran. 


\section{Referencias}

A. System, "La nueva revolución del mundo conectado", 2018. [En línea]. Disponible en: https://accent- systems.com/es/nb-iot/. [Consultado: 17-dic-2018].

C. Rehbein, "Nueva tecnología para medidores de agua dará mayor información a los clientes.", 2017. [En línea]. Disponible en: https://www.publimetro.cl/cl/ noticias/2017/02/15/nueva- tecnologia-medidores-agua-dara-mayor-informacionclientes.html. [Consultado: 15-nov-2018].

M. Quishpe, "Telemetria O Telectura Para Medidores De Agua Potable En El Centro Del Dmq", INSTITUTO DE ALTOS ESTUDIOS NACIONALES, 2008.

BMETERS, “Dispositivi per reti LoRaWAN", 2018.

lagua, "Lectura remota de contadores con la solución innovadora SensusRF", 2017. [En línea]. Disponible en: https://www.iagua.es/noticias/espana/sensus/17/10/19/ lectura-remota-contadores-solucion-innovadora-sensusrf. [Consultado: 06-sep2019].

IBM, "Servicios Web". [En línea]. Disponible en: https://www.ibm.com/support/ knowledgecenter/es/SS7K4U_9.0.0/com.ibm.websphere.zseries.doc/ae/cwbs_ wbs2.html. [Consultado: 22-abr-2019].

A. R. García Núñez, "Restful: un caso de uso de gestión de bibliotecas", 2017. [En línea]. Disponible en: http://oa.upm.es/45203/12/TFG_ADOLFO_RODRIGO_GARCIA_ NUNEZ.pdf. [Consultado: 02-nov-2019].

RedHat, "Cloud Computing". [En línea]. Disponible en: https://www.redhat.com/es/topics/ cloud-computing/what-is-private-cloud. [Consultado: 25-sep-2019].

"Cloud Computing - Aplicaciones en un solo tacto". [En línea]. Disponible en: https://www. salesforce.com/mx/cloud-computing/. [Consultado: 26-sep-2019].

D. G. IONOS, "laaS", 2019. [En línea]. Disponible en: https://www.ionos.es/digitalguide/ servidores/know-how/que-es-iaas/. [Consultado: 17-oct-2019].

S. Labs, "KanbanTool". [En línea]. Disponible en: https://kanbantool.com/es/. [Consultado: 13-nov-2019].

M. Naylamp, "Sensor de flujo de agua 1/2" YF-S201". [En línea]. Disponible en: https:// naylampmechatronics.com/sensores-liquido/108-sensor-de-flujo-de-agua-12yf-s201.html. [Consultado: 18-sep-2019].

B. Studios, "Balsamip". [En línea]. Disponible en: https://balsamiq.com/wireframes/. [Consultado: 11-may-2020]. 


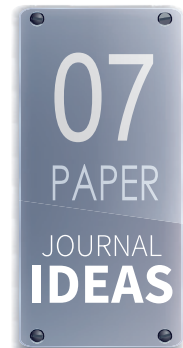


